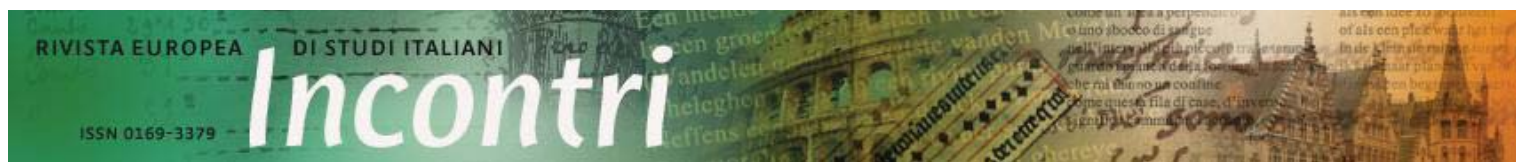

URN:NBN:NL:Ul:10-1-114277 - Publisher: Igitur publishing

Content is licensed under a Creative Commons Attribution 3.0 License

Anno 28, 2013 / Fascicolo 2 - Website: www.rivista-incontri.nl

\title{
La rappresentazione del potere cortigiano nel Marescalco di Pietro Aretino ${ }^{1}$
}

\section{Andrea Polegato}

Pietro Aretino (1492-1556), commediografo, autore satirico e poeta, venerato e temuto da papi e imperatori, scrive Il Marescalco nel 1526-27, a cavallo tra la deludente esperienza alla corte papale e il lungo (e fortunato) periodo veneziano. Giocando, infatti, sulla presunta sodomia del protagonista e sulle opinioni comuni a proposito del matrimonio e delle donne, Aretino sviluppa una satira feroce contro le corti dell'epoca (non solo quella mantovana, come dimostreremo) e contemporaneamente un panegirico di Venezia e della sua aristocrazia. Ricostruendo il meccanismo di funzionamento dell'ironia nella commedia, il presente articolo intende far emergere la personalissima critica dell'autore del Marescalco al potere cortigiano.

\section{Pietro Aretino tra Roma e Venezia}

Aretino nasce ad Arezzo e si trasferisce a Roma nel 1517, dove si procura fama e successo come autore di 'pasquinate', poemetti satirici scritti sulla base di anonime proteste contro la Curia. ${ }^{2}$ Il successore di Leone X al soglio di Pietro, Adriano VI, si mostra meno tollerante della spregiudicatezza di Aretino. Per questa ragione il commediografo è costretto a lasciare Roma per poi ritornare solo con l'elezione di Clemente VII (1523). Tuttavia, la sua intemperanza gli procura grandi nemici che giungono persino ad attentare alla sua vita. A questo si aggiunge il clamore suscitato dalla pubblicazione dei sedici Sonetti lussuriosi che lo costringe a lasciare Roma definitivamente. Nell'ottobre 1525, passa pertanto a servizio del condottiero Giovanni delle Bande Nere, fino alla morte di quest'ultimo (30 novembre 1526). Successivamente, dal dicembre 1526 al marzo 1527, Aretino soggiorna a Mantova presso l'allora marchese Federigo Gonzaga (poi Duca, a partire dal 1530); lì compone la prima versione della

\footnotetext{
${ }^{1}$ Le idee contenute in questo articolo sono la rielaborazione di due comunicazioni orali: la prima dal titolo 'L'ironia del Marescalco tra omosessualità e potere' presentata in occasione del Graduate Student Organization Colloquium, il 12 Maggio 2007 e ospitato dall'Università di Chicago; il secondo dal titolo 'Sexuality and Education in Pietro Aretino's Comedy The Stablemaster (Il Marescalco)' per il XVI ${ }^{\circ}$ Annual Newberry Library Center for Renaissance Studies Graduate Student Organization Colloquium, avvenuto il 23 Gennaio 2009 presso la Newberry Library, sempre a Chicago. Desidero ringraziare il professor Massimo Scalabrini dell'Indiana University, che mi ha introdotto allo studio di questo argomento, e il professor Justin Steinberg dell'Università di Chicago, che mi ha suggerito di insistere sull'ambiguità con cui Aretino dipinge la sessualità del protagonista della commedia.

${ }^{2}$ Cfr. G. Ferroni, 'Pietro Aretino e le corti', in: Pietro Aretino nel cinquecentenario della nascita. Atti del Convegno di Roma - Viterbo - Arezzo - Toronto - Los Angeles, Roma, Salerno, 1995, vol. I, p. 24.
} 
commedia /l Marescalco. ${ }^{3}$ Si trasferisce infine a Venezia dove, libero da ogni servitù cortigiana, pubblica il Marescalco e la Cortigiana, commedia scritta durante il tumultuoso periodo romano, nel $1534 .^{4}$ Come osserva Larivaille, non deve essere stato per caso se Aretino sceglie di pubblicare la Cortigiana per seconda, presentando quest'ultima le corti e i Signori in toni decisamente più irriverenti di quelli usati nel Marescalco. ${ }^{5}$ La nuova vita che Aretino intraprende a Venezia si ripercuote, infatti, anche sulle proprie scelte letterarie. In cerca di un pubblico più internazionale torna alla commedia solo nel 1542, facendosi conoscere e apprezzare grazie a lavori di altro genere, quali: i Ragionamenti, un rovesciamento dissacrante del Cortegiano; una lunga serie di scritti religiosi e agiografici, con i quali Aretino cerca di 'conferire alla propria vita un'onorabilità di buona lega'; e le Lettere, la cui elaborazione del tutto originale innalza l'autore a 'secretario del mondo'. ${ }^{6}$

\section{Sinossi e particolarità della commedia}

La commedia del Marescalco si apre con l'apparente elogio del Principe e il parallelo biasimo del marescalco, cortigiano e risaputo omosessuale, che crede di dover sposare per ordine del proprio Signore, il duca di Mantova, una bella fanciulla dalla dote cospicua. Recita l'Istrione al quale il prologo è affidato:

il magnanimo Duca di Mantova, essempio di bontà e di liberalità del nostro pessimo secolo, avendo un marescalco ritroso con le donne, come gli usurai con lo spendere, gli ordina una burla, per via de la quale gli fa tôr moglie con nome di quattro milia scudi di dota, e strascinatolo in casa del gentilissimo Conte Nicola, albergo di vertù e rifugio de i vertuosi, sposa per forza un fanciullo che da fanciulla era vestito. E scopertosi lo inganno, il valente uomo ne ha più allegrezza nel trovarlo maschio che non ebbe dolore credendolo femina. Ora se si pecca mortalmente a non dare un cavallo a quel venerabile castrone, che non ha paura d'essere un cujum pecus [.....$^{7}$ (Prologo)

In un solo paragrafo, Aretino concentra tutti gli elementi della commedia che vuole rappresentare: la sessualità del protagonista; la beffa ordita dal Duca sotto forma di matrimonio; l'agnizione finale; e l'ambientazione cortigiana. Alla sessualità del

\footnotetext{
${ }^{3}$ Purtroppo questa prima versione del Marescalco è andata perduta e il testo in nostro possesso è quello rimaneggiato da Aretino in occasione della sua pubblicazione a Venezia nel 1534. Sulla datazione del Marescalco e i problemi relativi alla prima e seconda versione si veda P. Aretino, Il Marescalco, in: Teatro, a cura di Rabitti, Boccia \& Garavelli, tomo II, Roma, Salerno, 2010, pp. 11-21 e G. Da Pozzo, 'L'Aretino, il "Marescalco", e i cavalli', in: Medioevo e Rinascimento veneto, con altri studi in onore di Lino Lazzarini, a cura di A. Limentani, Padova, Antenore, 1979, pp. 148-151. Da Pozzo ricostruisce anche il contesto storico della commedia dell'Aretino, compreso l'atteggiamento ambiguo del Gonzaga nei confronti di Giovanni delle Bande Nere (ivi, pp. 146-147).

${ }^{4}$ Secondo Larivaille la prima edizione del Marescalco, benché datata 'del mese di febraro' 1533, è stata in realtà pubblicata nel febbraio dell'anno successivo, pochi mesi prima della Cortigiana. Aretino, Il Marescalco, cit., p. 11.

${ }^{5}$ Ivi, p. 15

${ }^{6}$ Ivi, pp. 14-15. Per un approfondimento sulla vita e le opere di Aretino si rimanda a: P. Larivaille, Pietro Aretino fra rinascimento e manierismo, Bulzoni, Roma, 1980; per la sua produzione teatrale, M. Baratto, 'Commedie di Pietro Aretino', in: Idem, Tre saggi sul teatro, Venezia, Neri Pozza, 1964, pp. 69-155. Il rapporto tra Aretino e le Corti italiane è invece discusso da Ferroni, 'Pietro Aretino e le corti', cit., pp. 2348.

${ }^{7}$ Inoltre, i riferimenti al testo della commedia, tratti dall'edizione critica della già citata Salerno Editrice, saranno indicati in parentesi con l'atto in numeri romani e in numeri arabi la scena e il paragrafo (quest'ultimo secondo la divisione proposta da Petrocchi, edizione Mondadori, e accolta nell'edizione della Salerno).
} 
protagonista si allude ripetutamene: (1) nel rapporto con le donne, 'ritroso con le donne, come gli usurai con lo spendere' (2) nell'adesione contro voglia prima all'ordine del Duca, 'sposa per forza', e poi al sollievo provato nello scoprire la vera identità della sposa; e (3) negli appellativi dati al Marescalco, 'venerabile castrone', 'cujum pecus', ovvero bestione, e lo stesso 'marescalco', non un nome proprio (con cui al contrario molti altri personaggi sono identificati, per esempio: Giannicco, Carlo, Ambrogio, ecc.) ma la sua professione, stalliere di corte, dedito ai cavalli e alle bestie. In posizione almeno apparentemente antitetica al Marescalco, viene omaggiato il Duca di Mantova: 'esempio di bontà e di liberalità del nostro pessimo secolo'. Ma anche tale omaggio si presenta ambiguo: non è infatti chiaro se il Duca con il suo esempio confermi il 'pessimo secolo' o piuttosto ne rappresenti l'eccezione. ${ }^{8}$ Infine, il tema del matrimonio è presentato in una prospettiva meramente economica, e quindi comica: 'moglie con nome di quattro mila scudi di dota'.

L'agnizione, la sodomia, e la discussione su vantaggi e svantaggi del matrimonio costituiscono dei topoi tradizionali della commedia rinascimentale, ma nell'opera di Aretino essi vengono 'svuotati'. ${ }^{9}$ Innanzitutto, il pubblico viene informato da subito della sorpresa finale che attende il Marescalco che si ritrova sposato ad 'un fanciullo, che da fanciulla era vestito'. L'agnizione non potrà più essere svelata nel corso della narrazione privando così la commedia di uno degli espedienti più efficaci che ne ravvivano di solito lo svolgimento diegetico. ${ }^{10}$ Tale scelta ha una duplice funzione. Da un lato, sembra confermare le illazioni dell'Istrione sulle preferenze sessuali del protagonista il quale, per l'appunto, scoperta la vera identità della sposa 'ha più allegrezza nel trovarlo maschio' che nel trovarlo 'femina'. Dall'altro, permette di spostare l'attenzione del pubblico dalla 'ritrosia' del Marescalco ad assecondare l'ordine del Duca ai tentativi per convincerlo da parte degli altri cortigiani. Questi ultimi, al contrario del pubblico, sono gli unici a non conoscere davvero la natura burlesca dello sposalizio. ${ }^{11}$ Un'ultima particolarità della commedia è che la vicenda, pur coinvolgendo personaggi e ambienti cortigiani, si consuma in città, all'ombra dal 'Castello' e dal suo 'Signore': né la corte,

\footnotetext{
8 L'ambiguità dell'omaggio al Signore di Mantova si ripresenta anche all'interno della commedia. Il cortigiano Ambrogio commentando l'opinione positiva che Jacopo, altro cortigiano, ha di Federico Gonzaga, sembra essere concorde: 'tu parli da savio, ma non sarebbe di Gonzaga, se non fosse buono, umano e liberale' (III, 9). Tuttavia, i due avevano affermato in precedenza: Ambrogio, 'Sempre i signori fanno bene a chi no 'l merita, o a chi no 'l conosce'; Jacopo, 'I signori fanno de le altre cose più triste' (I, 3). La contraddizione è evidente e finisce per sminuire i loro elogi. Inoltre, nella scena precedente Ambrogio in un soliloquio si scaglia contro le corti e i suoi padroni (III, 8, 1).

9 Sul matrimonio, questione ampiamente dibattuta nel corso del Rinascimento, si veda, R. Leushuis, Le mariage et l' 'amitié courtoise' dans le dialogue et le récit bref de la Renaissance, Firenze, Olschki, 2003, p. 48 e nel caso specifico del Marescalco M. Sherberg, 'Il potere e il piacere: la sodomia del Marescalco' in: La rappresentazione dell'altro nei testi del '500, a cura di S. Zatti, Lucca, Pacini Fazzi, 1998, pp. 102-103.

${ }^{10} \mathrm{Nel}$ mancato sfruttamento dell'agnizione Ferroni vede una 'continua svalutazione [...] svuotamento e rovesciamento' del meccanismo comico, G. Ferroni, 'La costrizione al teatro: il Marescalco', in: Idem, Le voci dell'Istrione. Pietro Aretino e la dissoluzione del teatro, Napoli, Liguori, pp. 71-72, nota 1.

${ }^{11}$ Tra i personaggi che partecipano direttamente al dramma del protagonista, solo Giannicco, il garzone del Marescalco, e Ambrogio sembrano avere una qualche percezione che il matrimonio sia in realtà una burla, pur non sapendolo per certo: per il primo (II, 4) e (III, 3, 1); e per il secondo (III, 9, 1). Non a caso, Giannicco e Ambrogio si differenziano dagli altri personaggi in scena: il primo, intelligente quanto impertinente, si prende gioco del Pedante, ordendo ai suoi danni una beffa nella beffa (II, 1 e 2); il secondo è l'unico tra i cortigiani ad esprimersi contro l'ordine del Duca denigrando il matrimonio e le mogli e tessendo l'elogio di Venezia $(\mathrm{V}, 2)$.
} 
intesa come luogo fisico, né il Duca compaiono mai. ${ }^{12}$ Tutti questi elementi giocano un ruolo importante nella definizione del potere cortigiano e sono tra loro collegati. Partiamo pertanto dall'analisi della sessualità del protagonista.

\section{Le allusioni alla sessualità del Marescalco}

L'Istrione apostrofa il Marescalco con epiteti bestiali, comunemente attribuiti ai sodomiti, che precisano il senso della ritrosia del protagonista nei confronti delle donne. ${ }^{13}$ Il pubblico è infatti indotto a ritenere il Marescalco un sodomita e questa diventa la ragione apparente della sua riluttanza al matrimonio come della beffa stessa. Anche gli altri protagonisti della commedia condividono questa opinione, associando spesso il Marescalco alle bestie. Il suo garzone, per esempio, dice: '(p)ure si dice che voi siete una bestia, padrone, a non tor (moglie)' (III, 3); e così, Messer Jacopo, che riferendosi alla cocciutaggine del Marescalco nel rifiutare l'ordine del Duca, commenta: 'che bestiaccia! Mi par così vedere che questa pratica lo farà cacciare in malora' (I, 2). La Balia, unico protagonista femminile dell'opera, descrivendogli un sogno in cui un uomo molesta un uccello, lo interpreta così: '(l)'uccellino che cantava è il tuo ragazzo (Giannicco), che dolcemente ti ragionava de la moglie, l'uomo bestiale sei tu', per poi esortarlo ad abbandonare le sue 'gioventudini' (I, 6). Questi passaggi, che fornisco a puro titolo di esempio, sottolineano come tutti alludano alle pratiche sessuali del Marescalco e che pertanto quest'ultime siano viste come la vera causa della sua ritrosia. Tuttavia, a ben vedere, non c'è un solo passo della commedia dal quale si possa inferire con sicurezza che il Marescalco sia effettivamente un sodomita. ${ }^{14}$ Inoltre, nessun personaggio, tranne forse la Balia, sembra davvero interessato alla questione, tant'è vero che né la sua cocciutaggine né una sua ambigua quanto maldestra ammissione ad un passo d'altare $(5,10,3)$ impediscono la celebrazione della cerimonia nuziale voluta dal Signore. Per questa ragione, il centro dell'azione non è tanto la sessualità del Marescalco, che resta indeterminata e ininfluente, quanto la campagna di convincimento perseguita con altrettanta cocciutaggine dagli altri personaggi affinché egli assecondi il volere del Signore di Mantova. Da questi tentativi emerge, infatti, la natura del potere che il Duca esercita su tutti i cortigiani. ${ }^{15}$

\section{Il rapporto tra cortigiani e la natura del potere del Duca}

Nonostante la risaputa bestialità del Marescalco, tutti i cortigiani si prodigano per convincerlo a sposarsi. Essi giustificano questi sforzi in nome dell'amore e dell'amicizia che dicono di nutrire nei suoi confronti. Tra questi i più attivi sono il Conte e il Cavaliere, i quali più volte affrontano il protagonista:

\footnotetext{
${ }^{12} \mathrm{Ci}$ sono solo tre occorrenze nella commedia in cui alcuni personaggi accennano al Castello come luogo al di fuori della scena (II, 8; III, 5; III, 9).

${ }^{13}$ Sulle connotazioni bestiali della sodomia nel Cinquecento e non solo si veda Sherberg, 'Il potere e il piacere', cit., p. 109, nota 6. Per quanto riguarda la percezione della sodomia a Venezia, città in cui II Marescalco viene pubblicato, si rimanda a G. Ruggiero, The Boundaries of Eros: Sex Crime and Sexuality in Renaissance Venice, New York-Oxford, Oxford University Press, 1985, pp. 109-145.

${ }^{14}$ Come osserva Sherberg, persino sulla sua 'ritrosia con le donne', con cui l'Istrione lo presenta per la prima volta al pubblico, il Marescalco dimostra in realtà di avere invise le mogli $(I, 2,2$ e III, 5, 1) e non già tutto il genere femminile del quale al massimo critica la volubilità e superficialità (II, 3; V, 2, 4-9). Cfr. Sherberg, 'Il potere e il piacere', cit., p. 98.

${ }^{15}$ Cfr. ivi, p. 104.
} 
CONTE: Maestro, (a) io ti vo' bene, et a gli amici si vuol dar sempre ottimi consigli. [...] Di' poi che io non te l'abbia detto; tu deveresti pur sapere ed avere inteso da ciascuno, che (b) non c'è se non un Duca di Mantova al mondo, e che solo egli fra prencipi dona, accarezza e fa grandi $i$ servitori, e non vesteno così i primi gentil'uomini del Papa, né de lo Imperadore, come vesti tu [...]. E vaglion più le amorevoli parole di sua Signoria, che i fatti de gli altri; e se la sua umanità non ci facesse ognuno compagno, non ardiresti stare in su 'I tirato di ciò che ti comanda.

CAVALIERE: Il Conte ti favella da vero amico. (IV, 3, 3-4) $)^{16}$

In questo passo, il Conte descrive, quasi in termini erotici, la logica dello scambio che caratterizza il rapporto Signore-cortigiani: il Signore 'accarezza' i propri sudditi provvedendo alle loro necessità come nessun altro principe e, di conseguenza, i cortigiani non stanno 'in su 'l tirato', cioè assecondano i suoi ordini. La logica dello scambio funziona ovviamente anche all'inverso, come avverte nello stesso dialogo il Conte rivolto al protagonista: 'se il Signore intende questa tua fantasticheria [...] ti caccerà, e basta' (ivi).

Anche Messer Jacopo cerca di convincere il Marescalco alle nozze e per essere più convincente, decide di portare con sé il figlio, a mo' di dimostrazione vivente di quanto i figli siano tra le gioie più grandi del matrimonio:

lo che ho tenuto lunga pratica con il Marescalco, non potrei, se ben volessi, tener collera seco, ché (a) invero egli è uomo gentile e merita d'essere amato; io [...] voglio [...] e con l'esempio, e con il testimonio di questo mio figliuol maggiore riconciliarmi seco, e costringerlo a torla (la promessa sposa) per amore, a ciò che non gli fosse fatta tor per forza, non gne ne avendo poi né grado, né grazia. $(\mathrm{V}, 1)$

Terminato l'elenco di benefici che si hanno nell'avere un figlio, si rivolge al Marescalco retoricamente:

Et avendo [...] tu un (figlio) simile, (b) non lo averesti caro, come hanno $i$ vertuosi la liberalità del nostro Signor Duca? $(\mathrm{V}, 1)$

Tutti e tre i personaggi si interessano alla sorte del protagonista per amore e amicizia. ${ }^{17}$ Confrontando queste due scene emerge anche un altro elemento di continuità. In entrambi i dialoghi, infatti, si ripete la stessa struttura che nelle citazioni ho contrassegnato con le lettere (a) e (b). Ad ogni momento (a), nel quale si riafferma l'amicizia e la stima nei confronti del protagonista, corrisponde un momento (b), in cui ad essere lodato è il Gonzaga. I cortigiani accompagnano ogni considerazione sul Marescalco alle virtù e magnanimità del Duca. In altre parole, il rapporto di amicizia tra cortigiani è sempre vissuto all'ombra del Signore. Questa prospettiva è confermata dal Conte il quale dice del Duca: 'se la sua umanità non ci facesse ognuno compagno, (tu) non ardiresti stare in su 'I tirato di ciò che ti comanda'. Il significato immediato del passo è evidente: se il Signore non fosse tanto magnanimo da trattarci tutti alla stessa stregua tu non oseresti contraddirlo. Tuttavia significa anche che è l'umanità del Duca a

\footnotetext{
${ }^{16}$ Tutti i corsivi nelle citazioni dell’opera sono miei.

${ }^{17}$ Se Jacopo appare più sincero del Conte e del Cavaliere nel dichiarare la propria stima ed amicizia verso il Marescalco si deve anche alla gerarchia dei ruoli a corte. Il Conte e il Cavaliere appartengono infatti alla parte alta della gerarchia cortigiana, più vicini al Signore, mentre Messer Jacopo si trova collocato più in basso ma anche più vicino al ruolo occupato dal Marescalco. Per un'analisi sulla gerarchia nella commedia si veda Da Pozzo, 'L’Aretino', cit., pp.158-163.
} 
rendere i personaggi della commedia 'compagni'. Infatti, di fronte al rischio che la magnanimità del Signore venga meno, si dissolve anche il rapporto diretto tra cortigiani. È il caso del Conte e del Cavaliere i quali, all'ennesimo rifiuto del Marescalco, reagiscono sostituendo all'amicizia le minacce:

CONTE: [...] Riferiremo la tua asinaria al Signore; e s'egli ci commette che ti caviamo gli umori del capo, faremo il debito.

CAVALIERE: Tu fusti sempre un cavallo, e s'egli stesse a me, ti tratterei da quel che sei. (IV, 3, 8)

Il cambio di strategia da parte dei due cortigiani rivela la pretestuosità dello stesso tema matrimoniale. La ragione per cui i due personaggi tentano a più riprese di convincere il Marescalco non risiede nella bontà del matrimonio o nell'amicizia per il protagonista, ma nell'esecuzione degli ordini del Duca e nei vantaggi personali che da questa derivano. ${ }^{18}$ Quando qualcuno 'si tiene in sul tirato' con il Duca, come nel caso del suo marescalco ritroso, si rompe il rapporto sia con il proprio Signore che con gli altri cortigiani.

Per questa ragione, anche gli insulti che il Conte e il Cavaliere lanciano contro il Marescalco assumono qui una veste nuova. Mentre il pubblico, istruito dall'Istrione circa le pratiche omoerotiche del protagonista, collega questi epiteti alla sua sessualità, essi descrivono anche la riluttanza del protagonista ad assecondare l'ordine del Duca. Il Conte e il Cavaliere, infatti, contrappongono la bestialità del Marescalco all'umanità del Duca e della sua corte. Pertanto, da un lato, ci sono i cortigiani legati al loro Signore da una logica dello scambio sempre descritta in termini erotici: il Duca che 'dona, accarezza [...] i propri servitori'; i cortigiani che vogliono aiutare il protagonista perché lo amano; lo stesso Marescalco che all'inizio interpreta lo scherzo ordito dal Signore come 'segno d'amore' (I, 6). Dall'altro, ci sono il protagonista e la sua sessualità animalesca che lo pone al di fuori della corte in quanto ritroso, refrattario ad accettare l'amore (e gli ordini) del Signore. Ed è su questa dicotomia che la satira di Aretino si dispiega pienamente, ribaltando le parti.

\section{La corte e le donne}

In un dialogo con Giannicco, anche la Balia, come quasi tutti i cortigiani, elogia il Signore di Mantova. La donna aggiunge che il Duca, nel dar moglie al Marescalco, fornisce un esempio positivo a tutti quei 'ghiottoni' che per le loro ribalderie chiaramente di natura sessuale - dovrebbero essere arsi al rogo. ${ }^{19}$ Giannicco, forse perché colto sul vivo, essendo stato presentato dall'Istrione alla fine del prologo proprio come 'ghiottone', interviene su questo punto avvisando la Balia che sarà lei ad essere balzata, ovvero beffata:
G.: Voi sarete balzata.
B.: Chi mi balzerà?
G.: Tutta la corte.
B.: Perché?

\footnotetext{
${ }^{18} \mathrm{Cfr}$. Sherberg, 'Il potere e il piacere', cit., p. 103.

${ }^{19}$ Vale la pena notare che a Venezia erano chiamati 'cortigiani' proprio 'i ghiotti' insieme agli 'sviati, i ladroncelli, gli sbricchi e simili taglia-borse', come lo stesso Aretino fa dire a Nanna, protagonista del Dialogo nel quale la Nanna insegna a la Pippa, pubblicato nel 1536. P. Aretino, Sei giornate: Ragionamento della Nanna e della Antonia (1534); Dialogo nel quale la Nanna insegna a la Pippa (1536), a cura di G. Aquilecchia, Bari, Laterza, 1969, p. 234.
} 


\section{G.: Perché è nimica de le donne. (II, 4)}

Non è la prima volta che il ragazzo sembra conoscere o intuire il finale a sorpresa che attende il Marescalco ma qui, contestando le affermazioni della Balia, dimostra ancora una volta che il matrimonio non serve a sanare una situazione moralmente riprovevole ma semmai a confermarla, essendo un matrimonio tra due uomini. Inoltre, la motivazione con cui Giannicco giustifica l'avvertimento ai danni della Balia getta una nuova luce sul contrasto corte-Marescalco che abbiamo definito. Il matrimonio non può avere alcun valore esemplare perché 'tutta la corte' è misogina, 'nimica delle donne'. La giustificazione del garzone è interessante anche per il rimando testuale in essa contenuto.

Nella Calandria di Bernardo Dovizi da Bibbiena (1470-1520) ritroviamo, infatti, la stessa caratterizzazione della corte. Lidio, protagonista della commedia, e il servo Fessenio incontrano Polinico, pedagogo di Lidio e prototipo del personaggio del Pedante di Aretino. Polinico cerca di dissuadere Lidio dall'amore che prova per una donna romana. Gli argomenti adotti dal pedagogo sono ancora una volta la volubilità e infedeltà delle donne. Fessenio però lo interrompe accusandolo di conformarsi 'al viver d'oggi'. Incautamente il pedagogo chiede in quale modo e così risponde il servo:

FESSENIO: Nell' essere inimico delle donne, come è quasi ognuno in questa Corte la corte romana]: e però ne dici male. $E$ iniquamente fai.

LIDIO: Dice il vero Fessenio: perché laudar non si può quel che tu hai detto di loro, per ciò che sono quanto refrigerio e quanto bene ha il mondo, e sanza le quali noi siamo disutili, inetti, duri e simili alle bestie.

F.: Che bisogna dir tanto? Non sappiàn noi che le donne sono sí degne che oggi non è alcuno che non le vadi imitando e che volentieri, con l'animo e col corpo, femina non diventi? $(I, 2)^{20}$

Come la corte mantovana descritta da Giannicco, anche quella romana ha invise le donne e, tuttavia, tutti le imitano (e volentieri) nell'animo e nel corpo. Il discorso di Giannicco si accorda perfettamente a quello di Fessenio, non solo perché la corte mantovana ha nei confronti delle donne lo stesso pregiudizio ma anche perché, come alla corte romana, Duca e cortigiani si comportano come donne, nell'animo e nel corpo.

In un dialogo con lo Staffiere di corte, il Marescalco già sull'orlo della disperazione alla prospettiva di doversi sposare, così descrive il proprio Signore:

lo credo a Dio, e questi Signori hanno di strani capricci [...]. Se io volessi moglie col dotarla del mio, e ricercassi il suo favore per mille mezzi e con centomila supplicazioni, non l'averei mai; e perché io non la voglio, me la vuol dar per forza: eglino sono come le donne, le quali corrono dietro a chi le fugge, e fuggono chi le seguita, e non hanno altro piacere che far disperarle $i$ poveri servitori. (II, 3 )

Se il comportamento del Duca viene associato alla volubilità femminile, anche quello che i cortigiani assumono al suo cospetto non è da meno. Il Pedante, che al contrario del Marescalco non nasconde le proprie preferenze sessuali per i suoi discepoli, descrive il proprio rapporto con il Signore in termini persino più espliciti di quelli che abbiamo visto utilizzare dal Conte: ${ }^{21}$

\footnotetext{
${ }^{20}$ B. Dovizi, La Calandria, a cura di G. Padoan, Padova, Antenore, 1985, p. 76. I corsivi sono miei.

${ }^{21}$ Che il Pedante sia un sodomita (attivo e passivo) è provato da numerosi passaggi ben più espliciti di quelli sul e del Marescalco, se non fosse altro che a pronunciare frasi a doppio senso o esplicite dichiarazioni è il
} 
Ne le intestine, ne le viscere, ne lo utero mi hanno penetrato le accoglienze che mi ha fatto sua eccellentissima Signoria. (III, 10)

Come in una penetrazione anale - 'intestine', 'viscere', e 'utero' nelle parole del Pedante - il Signore agisce da soggetto attivo mentre il cortigiano da attore passivo. L'immagine del potere cortigiano che ne scaturisce è quella di un Duca capriccioso le cui voglie sono ben volentieri assecondate dai suoi cortigiani.

Come si vede, le idee espresse da Fessenio nella Calandria trovano puntuale conferma nel Marescalco e ci permettono di estendere la critica di Aretino alla corte di Mantova anche a quella romana, che l'autore aveva abbandonato pochi mesi prima. ${ }^{22} \mathrm{Si}$ comprende ora una delle ragioni per cui il Duca è assente dalla scena teatrale. Lo zelo con cui quasi tutti i cortigiani eseguono i suoi ordini e desideri non rende la sua presenza necessaria. Inoltre, la sua assenza, come quella della Corte, intesa come luogo fisico, contribuisce a liberare la commedia da una costrizione geografica: data la natura del potere cortigiano, essa potrebbe aver luogo in qualsiasi altra corte, quella romana per prima. ${ }^{23}$

\section{Conclusioni}

Nel prologo l'Istrione presenta al pubblico la ritrosia del Marescalco come la causa attorno a cui ruota tutta la commedia. La sua caparbietà nel non accettare la sposa offertagli dal Duca viene da subito imputata alle sue preferenze sessuali. Tuttavia, come abbiamo dimostrato, le sue presunte pratiche sessuali non trovano mai conferma definitiva sulla scena né la sua caparbietà serve davvero ad evitare la cerimonia nunziale. Inoltre, l'agnizione finale svuota di significato la stessa questione matrimoniale in quanto la scoperta che la promessa sposa è in realtà un ragazzo non sana una situazione moralmente riprovevole ma semmai la assolve. Se la sessualità del Marescalco e il matrimonio risultano del tutto ininfluenti agli esiti della commedia, ne consegue che il bersaglio polemico della beffa è un altro. Il centro della commedia, infatti, è strutturato, sin dal prologo, dalla campagna di convincimento organizzata dagli altri cortigiani che autonomamente, cioè lontano dal Duca e dal suo Castello, si profondono volentieri nel tentativo di persuaderlo ad assecondare l'ordine del loro Signore. La ritrosia del Marescalco a sposarsi viene vista come un atto di ribellione nei confronti del Duca e quindi giudicata perversa tanto quanto la sua sessualità. Alla fine però è l'accomodarsi alla volontà del Principe a coincidere con un vero e proprio atto di sottomissione sessuale che coinvolge tutti. Per questo, la beffa colpisce tutti $i$ personaggi in scena e non già il solo Marescalco: ${ }^{24}$

Pedante stesso; si vedano a titolo di esempio: II, 1, 2 e 9; III, 10. Sulla figura del pedante come personaggio comico rimando a A. Stäuble, 'Parlar per lettera'. II pedante nella commedia del Cinquecento e altri saggi sul teatro rinascimentale, Roma, Bulzoni, 1991.

22 Il riferimento testuale alla Calandria non costituisce la sola menzione fatta da Aretino alla corte romana e alla propria esperienza personale a Roma, si veda per questo: II, 5 e IV, 3.

${ }^{23}$ L'unico personaggio a schierarsi dalla parte del Marescalco ritroso è Ambrogio, che dopo un pallido tentativo di convincerlo a sposarsi, finisce per fornire un resoconto dettagliato degli svantaggi che si hanno a prender moglie. Non è allora un caso che Aretino faccia pronunciare proprio a questo personaggio l'elogio di Venezia e dei suoi Signori (II, 5).

${ }^{24}$ Il fatto che tutti i protagonisti della commedia finiscano per essere vittime della burla ordita dal Duca non doveva stupire il pubblico veneziano. Infatti, è stato rilevato come nella Venezia rinascimentale ci fosse la tendenza, unica nel panorama europeo dell'epoca, a giudicare più riprovevole il ruolo giocato dal soggetto attivo di un rapporto omoerotico, in questo caso il Duca, che quello di chi vi si assoggettava, ovvero i suoi cortigiani. Dall'analisi, compiuta da Guido Ruggiero, dei processi condotti dalla Repubblica di Venezia 
CONTE: Adunque noi ci siamo stati?

CAVALIERE: Stati ci siamo, ah, ah, ah!

AMBROGIO: Ora sì, che ci potiamo chiamare babbioni mantovani, ah, ah, ah! $(\mathrm{V}, 10,8)$

Pertanto, se l'opera si apre con l'apparente elogio del Signore e il parallelo biasimo del suo marescalco, essa si evolve invertendo progressivamente le parti: ad un Marescalco che vive serenamente la propria sessualità - qualunque essa sia - e che, pertanto, non vuole accettare il matrimonio, si contrappongono cortigiani che vivono e si rapportano tra loro solo in una logica di mero scambio. Ė in questo quadro che l'ironia di Aretino centra il suo vero bersaglio: giocando sull'omosessualità del protagonista, a tutti nota e causa della burla stessa. La commedia finisce così per tratteggiare in modo preciso ma impietoso la reale natura dei rapporti tra cortigiani all'ombra del loro Signore, non solo alla corte di Mantova ma, grazie ad un felice rimando intertestuale alla commedia del Bibbiena, anche alla corte di Roma che Aretino aveva da poco abbandonato.

contro i sodomiti, emerge tale tendenza nelle condanne inflitte dai magistrati veneziani: 'the passive male was held to be less culpable for his acts and, therefore, normally escaped the death penality of his active partner' (G. Ruggiero, The Boundaries of Eros, cit., p. 145). Ciò dimostra ancora una volta quanto ambiguo sia l'elogio a Federico Gonzaga che Aretino fa recitare all'Istrione nel Prologo: 'esempio di bontà e di liberalità del nostro pessimo secolo'. 


\author{
Parole chiave \\ Pietro Aretino, Marescalco, potere, sodomia, corte, beffa
}

Andrea Polegato sta completando la tesi dottorato in letteratura italiana all'Indiana University, Bloomington. Si occupa di letteratura rinascimentale e filosofia politica. La tesi di dottorato tratta dei primi scritti amministrativi e diplomatici di Niccolò Machiavelli (1498-1512) con l'obbiettivo di definire il linguaggio politico in uso presso i funzionari della Repubblica di Firenze e il suo influsso sul pensiero machiavelliano maturo. Si interessa anche di comparazione tra Rinascimento italiano e Cina antica. Su questo tema, ha copubblicato un articolo che analizza i film 'Il mestiere delle armi' e 'Cantando dietro i paraventi' di Ermanno Olmi; ha inoltre ideato e insegnato un corso sull'arte della guerra che mette a confronto Machiavelli e il suo corrispettivo cinese, Sunzi.

1020 East Kirkwood Ave.

Ballantine Hall 642

Bloomington, IN 47405 (USA)

apolegat@indiana.edu

\title{
SUMMARY \\ Representation of Courtly Power in the Marescalco of Pietro Aretino
}

This article addresses a particular form of power, courtly power, displayed by Pietro Aretino in his play Il Marescalco (the Stablemaster). Aretino's perspective is particularly interesting because he is probably the only intellectual in fifteenth- and sixteenthcentury Italy who managed to emancipate himself from any political influences. From this privileged position, Aretino's play describes the relationship between the monarch and his courtiers and the real nature of the legitimization of power. Indeed, the character of the Duke of Mantua never appears on stage despite his role in organizing the prank against the protagonist, the Stablemaster. In this way, the audience's focus is mainly on the courtiers and their zeal in carrying out the Duke's orders. Aretino also chooses to set his play in the center of the city of Mantua rather than in the Court. The twofold absence of the Duke and the Court on stage underscores the real nature of courtly power. According to Aretino, its legitimacy lies primarily in the courtiers' passive attitude towards their ruler and in their attempts to favor him rather than in his direct intervention. Furthermore, while power is generally seen as a purely masculine domain, Aretino turns this idea upside-down in a very original way, by describing the passive attitude of the courtiers towards their master as a homosexual intercourse and the courtiers themselves as woman-haters. 